\title{
8. DISCUSSION FOLLOWING THE REPORT BY KADOMTSEV AND TSYTOVICH
}

(Wednesday, September 10, 1969)

\section{Chairman: S. A. Colgate}

Editor's remarks: The following discussion was long and chaotic, in spite of the energetic efforts of the Chairman. Apparently the subject was unfamiliar to many participants. I did quite some reshuffling. A long contribution by Ozernoi on photon whirls in the primeval Metagalaxy has been transferred to Chapter 13.

Kaplan: I would like to suggest some possible observations of plasma turbulence in the interstellar medium. Before I do this, it is necessary to note that all plasma wave modes are confined in relatively small regions of interstellar or circumstellar space, because only there the conditions are met for excitation of turbulence. Plasma waves cannot travel long distances. It is also very improbable that, in any given volume of the interstellar medium, the conditions for excitation of plasma turbulence will exist for a long time. Streams of charged particles, which are the most effective agent for producing high-frequency modes in the plasma turbulence, usually undergo a fast isotropization. The shock which can induce low-frequency turbulence passes through any given point in a short time. Plasma waves without continuing excitation decay soon by collisions or by Landau damping. I would therefore guess that plasma turbulence in the usually quiet interstellar medium will be encountered in small volumes and for short periods only, but quite frequently. I will call such volumes 'plasma-turbulence pockets'. Of course, in quasars and in pulsars, plasma turbulence is much more common; but here we are discussing the interstellar medium.

Can we see these 'pockets'? It is well known that Langmuir waves can easily be converted into electromagnetic waves of nearly the same frequency. This process explains many features of sporadic solar radio noise. But the Langmuir frequency in the interstellar medium is approximately $10^{4} \mathrm{~Hz}$ and cosmic radio noise of that frequency cannot be detected. Direct observations of this electromagnetic radiation are therefore impossible. However, there are some ways to observe the interstellar plasma turbulence pockets.

(1) Plasma waves with frequency $\omega_{p}$ can be transformed into electromagnetic waves with frequency $\omega \approx 2 \omega_{p}\left(c / V_{p}\right)\left(\varepsilon / m c^{2}\right)^{2}$ by scattering on relativistic cosmic-ray electrons. Here $V_{p}$ is the phase velocity of plasma waves, $c$ is the velocity of light, and $\varepsilon$ is the energy of the relativistic electrons. In this way, not only Langmuir waves but also very low-frequency ion-sound or magneto-sound waves can be transformed into electromagnetic waves with observable frequencies.

The radiation so produced has the same spectrum as the synchrotron radiation; and the intensities of both are comparable, if the energy density of plasma-wave 
turbulence is of the same order of magnitude as the magnetic energy density. However, since the pockets of plasma turbulence are rather small, the turbulent radiation is rarely seen against the continuous synchrotron background radiation in the Galaxy.

(2) The conditions for the observation of plasma-turbulence pockets are much better, if the relativistic electrons in these regions have an anisotropic velocity distribution; for example, if there are streams of relativistic electrons. The induced conversion of plasma waves into electromagnetic waves by scattering on the streams leads to an instability in the electromagnetic waves (interstellar maser-effect in continuous spectra). The amplitude of the electromagnetic waves increases until nearly all the energy of the stream of relativistic electrons is transformed into radiation. The plasma waves function as an ignition mechanism. In the ideal case of a very wellcollimated stream, the frequency of converted radiation is the same as mentioned sub (1), but more often it is of the order of $\omega \approx 2 \omega_{p} c /\left(\left\langle\theta^{2}\right\rangle V_{p}\right)$. Here $\theta$ is the angle between the electron velocity and the direction of the stream. The spectrum of this radiation also resembles the synchrotron radiation, and in addition there is almost complete polarization. Therefore, the interstellar plasma pockets in which the velocity distribution of relativistic electrons is anisotropic can be seen on the synchrotron background as bright spots with different polarization and with an intensity that is changing rapidly with the time because of isotropization of the stream.

(3) The anisotropy of relativistic particles and their concentrations may be increased by the turbulence pocket itself, since the plasma turbulence can serve as an effective accelerator of charged particles. But in this case, the output of electromagnetic energy is not so high, because the energy of the fast particles is taken from the energy of the plasma turbulence in the same pocket. I believe, incidentally, that most of the energy of the cosmic rays does not derive from the interstellar medium, but from violent events (supernovae).

(4) Under appropriate conditions, plasma turbulence pockets can enhance spectralline radiation of background ions and atoms and in this way form interstellar masers. I am not sure that this mechanism can really explain the anomalous $\mathrm{OH}$ emission, but the possibility deserves further study. I cannot give the details here, but refer you to a recent paper (Kaplan and Tsytovich, 1969a).

(5) When an electromagnetic wave passes through a plasma turbulence pocket, its frequency is changed by the non-linear fusion (coalescence) with plasma waves, $\left(\omega^{\prime}=\omega \pm \omega_{p}\right)$. This process is stochastic and therefore leads to an overall increase of the width of the emission line. In the paper cited above, the following formula has been established for the increase of the line width by Langmuir turbulence:

$$
\left\langle\left(\Delta \omega_{*}\right)^{2}\right\rangle=\frac{\omega_{p}^{4}}{\omega_{*}^{2}} \frac{W}{n_{e} m c^{2}} \frac{r}{\lambda_{p}} .
$$

Here $\omega_{*}$ is the frequency of the line, $\lambda_{p}$ is the wavelength of the plasma waves, $W$ is their energy density, and $r$ is the path length through turbulent regions. I shall use this formula for some estimates.

The observed emission lines of interstellar $\mathrm{OH}$ are very narrow. As a matter of fact 
the increase of their width due to turbulence in the interstellar medium cannot be more than $\Delta \omega_{*}=10^{2} \mathrm{~Hz}$. We have $\omega_{*}=10^{10} \mathrm{~Hz}$ and if we assume $\omega_{p}=10^{4} \mathrm{~Hz}$ and $n_{e}=0.1 \mathrm{~cm}^{-3}$, we have $W \lesssim 10 \lambda_{p} / r \mathrm{erg} \mathrm{cm}^{-3}$. The value of the ratio $\lambda_{p} / r$ is unknown, but we may estimate $\lambda_{p} / r \approx 10^{-18}$, so that $W$ is less than $10^{-17} \mathrm{erg} \mathrm{cm}^{-3}$. Of course, in small pockets the energy density of plasma turbulence may be much higher. Let me add that this process of fusion of electromagnetic and plasma waves leads to an apparent increase in the angular diameters of $\mathrm{OH}$ sources.

I would like to finish my remarks by referring to a review paper by Tsytovich and myself on the plasma mechanisms of radiation (Kaplan and Tsytovich, 1969b). (Kaplan, S. A. and Tsytovich, V. N.: 1969a, Astrofiz. 5, 21 ; Kaplan, S. A. and Tsytovich, V. N.: 1969b, Usp. Fiz. Nauk 97, 77.)

Colgate: Dr. Kaplan, you mentioned the width of an $\mathrm{OH}$ line. At what distance was this source? Can we estimate the probability of the existence of regions of at least lighter turbulence in our Galaxy?

Kaplan: I took the distance to the $\mathrm{OH}$ source as $300 \mathrm{pc}$ and assumed $\lambda=10^{3} \mathrm{~cm}$.

Syrovat-skii: I have a remark concerning the excitation of high-frequency plasma turbulence. To elucidate the question, consider the main channels of energy transformation in astrophysical situations (see Figure 1). It seems likely that the primary form of energy is the kinetic energy of macroscopic plasma motion. The causes of this motion may be nuclear burning, stellar explosions, gravitational contraction, rotation, convection, and stellar wind, as in the case of Earth's magnetosphere. These processes supply kinetic energy to the plasma motion and lead to the distortion and amplifica-

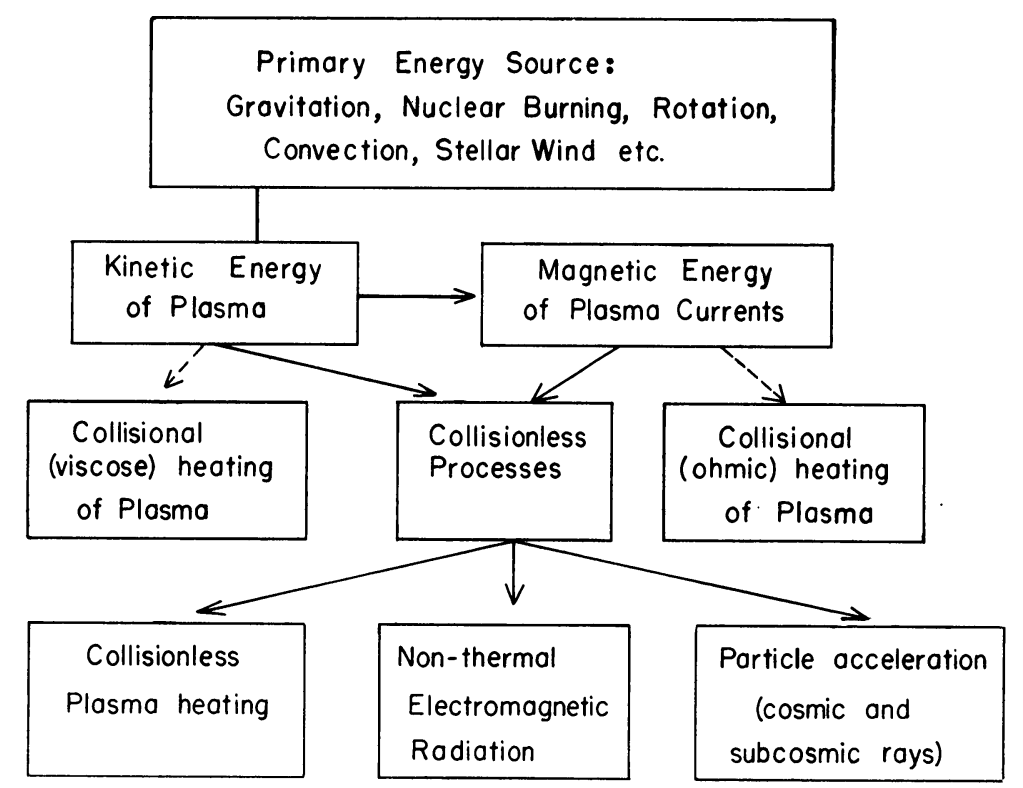

Fig. 1. (See the remark by Syrovat-skii.) Main channels of energy transformation in astrophysical situations. 
tion of the magnetic field. Because of the low collision rate between particles in a rarefied plasma, the dissipation by viscosity and finite conductivity is slow; and the main channel of energy transformation is seemingly collisionless dissipation through the excitation of plasma turbulence and acceleration of particles. As main causes of plasma turbulence, Kadomtsev mentioned the collisionless shock wave and the anisotropy of velocity distributions produced by magnetic contraction of a plasma.

I should like to add another process, which may be even more effective than shock waves. This is the formation of current sheets (see Figure 2). Such a sheet develops,

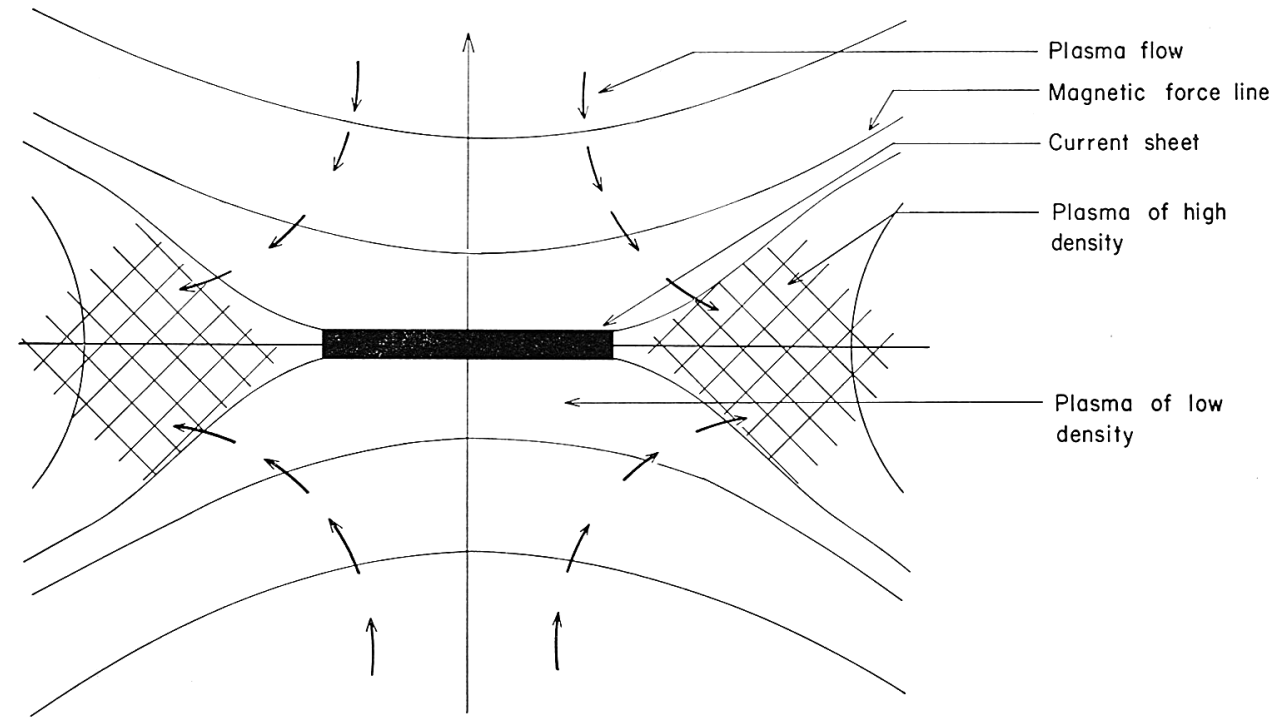

Fig. 2. (See the remark by Syrovat-skii.) Structure of a current sheet.

for example, near magnetic zero (neutral) lines as a result of plasma motion in its vicinity. The plasma flow to the current sheet can be calculated on the basis of magnetohydrodynamics. However, the essential feature of the sheet is its thickness, which is determined by microscopic parameters such as the particle gyroradius. It may be shown that the current density and, correspondingly, the directed electron velocity are large enough to excite plasma instability and turbulence, which lead to collisionless dissipation of the magnetic energy in the current sheet. This process is especially effective because the energy of neighboring, antiparallel magnetic fields is completely released in the small volume of the current sheet. It may be supposed that current sheet formation is a quite general property of non-uniformly moving magnetized plasma. In this case, the dissipation in the sheet is the main dissipative process.

Colgate: What is the rate of dissipation in such a current sheet?

Syrovat-skii: The rate of dissipation is fully determined by the velocity of the influx of magnetic energy into the sheet, which depends on the plasma flow at large distances.

Colgate: It seems to me that the dissipation in these current sheets is always limited 
by the rate at which the plasma in the sheet can emit the energy produced. In some cases, this may be only cooling by radiation; in others, it may be the emission of waves. Have you considered the ratio of the wave emission to heat emission in the form of light and X-rays? Which is more important?

Syrovat-skii: In quasi-stationary cases, the energy released in the sheet may be estimated using only the external parameters of the flow. The effective resistivity is such that it produces the required dissipation. It is a very complicated problem to calculate the ultimate energy losses.

Dubov: Dr. Syrovat-skii, I think you should place shock waves in a separate rectangle in your figure because it represents a different mode of energy dissipation.

Colgate: To me the difference between the current sheet and the shock wave is that in a shock wave the fluid passes through the discontinuity and has only a finite energy density behind the shock wave; whereas, in the current sheet, matter continuously, flows into a discontinuity, with no escape. Therefore a fundamental difference exists between the properties of a shock wave and of a current sheet composed of opposite magnetic fields. These different properties lead to rather different final conditions of the plasma.

Tsytovich: I have two comments. The first is about a possible mechanism of excitation of turbulence in the presence of non-thermal electromagnetic radiation. This particular mechanism is decay of an electromagnetic wave into another electromagnetic wave and a turbulent wave. We know that energy flows out from radio galaxies, quasars, and the like. From the observed output of radiation by such sources, it is possible to estimate the energy of the turbulence in the source. One finds that there is much less excitation of low-frequency turbulent motions than of highfrequency motions. The most important mode is excitation of Langmuir turbulence. From the amount of radiation one can estimate that in the case of quasars, for example, the energy of Langmuir turbulence must be of the order of $1 \mathrm{erg} \mathrm{cm}^{-3}$. It is of the same order of magnitude as the thermal energy of the particles. Therefore the turbulence must be very high in quasars. For supernovae, one can estimate that the energy of the turbulence from the observations is about $10^{-4}$ to $10^{-6}$ of the thermal particle energy, but much greater $\left(\sim 10^{4}\right.$ to $\left.10^{5}\right)$ than the thermal plasmon energy. For the interstellar medium the energy of the turbulence excited by the radiation is so low that it is much less than the thermal energy of the plasmons. Thus the radiation in the radio-frequency range cannot excite turbulence in the interstellar medium.

My other remark concerns the possible channels of the energy dissipation of the turbulence. Figure 1 of the Report by Kadomtsev and myself shows the dispersion of the various types of plasma waves. Because of non-linear effects, a high degree of isotropization exists. In isotropic turbulence the non-linear effects transform waves downward in frequency. Therefore the transfer of turbulent energy is downward along the curves of Figure 1 at p. 111. Consider first ion-sound turbulence. When $\omega=\omega_{B i}$, ion cyclotron damping occurs, which heats the ions. In the outer regions, there is Landau damping on electrons, and this damping heats the electrons. Thus two channels exist for the dissipation of the ion-sound turbulence. For Langmuir turbu- 
lence, the situation is different. Landau damping can occur only for large values of $k$, and for isotropic turbulence the energy transfer is away from the region of Landau damping. Therefore only collisional damping occurs in the region of small $k$. However, if the velocity distribution is anisotropic, or if some magnetic bottles exist, the direction of energy transfer can be reversed, and we will have a high rate of collective heating of electrons. For all low-frequency waves, efficient Landau damping on electrons occurs because the phase velocity is less than $V_{T e}$. Therefore low-frequency turbulent motion will heat the electrons and the plasma. Langmuir turbulence is better adapted for acceleration of particles, although it too shows Landau damping and produces the tail of the Maxwell distribution. In summary, the first channel of dissipation of turbulent energy is collective dissipation in the form of Landau damping or cyclotron damping. The second channel is collisional dissipation, similar to the channel in usual hydrodynamical turbulence. The third channel is particle acceleration and the fourth is radiation. Langmuir turbulence can be dissipated efficiently and transformed into radiation. For a certain critical dimension of the plasma all the energy of the turbulence goes into radiation.

Colgate: Rosenbluth and $\mathrm{McCrea}$ have been calculating the transformation of the Langmuir turbulence into the transverse mode of the electromagnetic radiation. The reason this transformation becomes so important is the following: the usual crosssection for scattering photons off electrons is the Compton cross-section. But when Langmuir turbulence is excited and plasma oscillations occur, then the cross-section is increased by a factor that can be as large as the number of electrons per cubic Debije length $\left(n_{e} \lambda_{D}^{3}\right)$. In addition, this factor is multiplied by $W_{k} /\left(n_{e} k T\right)$. For a supernova ejection, a total factor can result as large as $10^{18}$. Therefore one has to multiply the Compton cross-section by a very large number to get the cross-section for the scattering of electromagnetic radiation by the cooperative modes in a plasma oscillation. The result is a diffusion of photons in $k$-space; and just as in the preceding remark by Tsytovich, turbulence decay is represented by a diffusion of phonons in $k$-space.

Syrovat-skii: Tsytovich discussed emission of radiation by plasma turbulence. What about absorption? Do we have some indication of absorption of radio waves from discrete sources?

Tsytovich: Reabsorption of radio waves emitted by the source can be negative, i.e., the emitted waves can be amplified. The necessary condition for such a plasma maser effect is very easy to fulfill, and therefore the efficiency of the plasma mechanism of emission is very high. There is evidence of scattering of radar waves on high-frequency turbulence in the Sun. Will Dr. Gordon say a few words about the observations?

Gordon: I would like to discuss an example of nonlinear interaction of electromagnetic waves and plasma waves which occurs in the solar corona. My discussion is based on the study of radar echoes of the Sun carried out since 1961 by J. C. James at 38 and $25 \mathrm{MHz}$ (James, 1964, 1966). In the conventional theory the radar echo by the Sun is explained by the scattering of the beam in the vicinity of the layer where the refraction index tends to zero and the laws of geometrical optics are violated. In the framework of this theory the only parameters on which the effective cross-sections 
depend are the global distributions of the electron concentration $n_{e}$ and the electron temperature $T_{e}$. The variations in these distributions are small, even in the course of the cycle of solar activity. We therefore expect that the effective cross-sections will remain between 1 and 3 times $\pi R_{\odot}^{2}$. However, the observed radar echoes from the Sun behave quite differently. First, the effective cross-sections vary over a large range, from $\approx 100 \pi R_{\odot}^{2}$ down to a few times $10^{-2} \pi R_{\odot}^{2}$. Second, the frequency displacements have a symmetrical distribution of components, and if the radar reflections originate on inhomogeneities 'frozen' in the outflowing solar wind the displacements must be predominately to the 'violet'. A third difficulty is that reflections originating in very high layers of the corona, up to $3 R_{\odot}$ from the center, demand a 100 -fold increase of the local density, which is highly improbable even if we are dealing with strong shocks there. Also, on days when reflections are strong, sporadic radiation too is strong and fluctuating. This peculiar interdependence cannot be explained in the framework of the classical theory. But the results of the radar experiments can be explained self-consistently by the assumption that the reflected signal arises from the back-scattering from the turbulence excited in a coronal plasma situated above the plages (Gordon, 1968, 1969). The frequency displacements are therefore not Doppler shifts, but result from the induced processes of wave coalescence and decay of the type $t^{l} \rightleftarrows t+S$, where $t$ and $S$ represent photon and ion acoustic waves, respectively. The analysis of the reflected signal enables us to ascertain that the reflections do not arise in the quiet corona. (James, J. C.: 1964, IEEE Trans. Ant. Propag. AP-12, 876, 1966, Astrophys. J. 146, 356; Gordon, I. M.: 1968, Astrophys. Lett. 2, 49, 1969, Astrophys. Lett. 3, 181.)

Meyer: I would like to comment on the physical picture underlying these radar observations. H. U. Schmidt and I (Meyer and Schmidt, 1969) have investigated hydrodynamic flows along magnetic flux tubes in the low viscosity solar atmosphere. We considered flux tubes that arch back to the photosphere and found that very small pressure differences between the two footpoints must lead to the following characteristic flow pattern. The flow rises slowly at the base rapidly near the top of the flux tube and reaches the sonic velocity at the summit of the magnetic arch (or very near the summit, depending on the cross-sectional changes along the flux tube). The flow descends supersonically on the other side, passes through a shock front by which it adapts to the pressure condition on the low pressure side, and moves subsonically down to the other end. Since the viscosity in the solar atmosphere is small, even small pressure differences drive such flows. Such small pressure differences are to be expected between the two feet of the arched tube where the magnetic flux density is different, as will in general be the case.

On the basis of such a model one expects sonic and supersonic velocities along magnetic flux tubes that arch back to the solar surface. This situation is in striking contrast to all such flux tubes that lead into the solar wind. There the flow is limited by Parker's solar wind solution, which reaches the sound velocity only at the distance of several solar radii and accordingly leads to velocities of only 10 to $30 \mathrm{~km} \mathrm{sec}^{-1}$ in the corona. 
The suggestion that sonic and supersonic flows in magnetic arches are responsible for the features of the radar observations of the solar corona seems to be in general agreement with those observations. The disappearance of high-velocity signals above $1.5 R_{\odot}$ agrees with the indication from eclipse pictures that there are no magnetic arches above this level but only outgoing flux tubes, belonging to the solar wind. On these arches one will find both ascending and descending velocities up to and larger than the velocity of sound, 100 to $200 \mathrm{~km} \mathrm{sec}^{-1}$. The appearance of these flows depends on the existence of the magnetic arches, and the radar signals therefore will change with the rotation of the Sun and the solar activity. One might add that plage regions especially are regions of possible footpoints of such coronal magnetic arches. (Meyer, P. and Schmidt, H. U.: 1969, Z. Angew. Math. Mech. 48, T218.)

Field: If plasma turbulence is important in the interstellar medium, we are interested in the sources of such turbulence. Dr. Tsytovich, do you expect the sources of the turbulence to be localized around energetic sources such as supernovae explosions, shock waves from various sources, etc.? And if so, can this turbulence then propagate into the quiescent interstellar medium? Can it do so at all, particularly if Langmuir turbulence is involved, since plasma oscillations have a very small phase velocity?

Tsytovich: I think that the region in which plasma turbulence exists must be much larger than the sources of the turbulence itself, e.g., supernovae. Many processes exist that can transport turbulent energy: ejection of fast particles; high power radiation, and other processes.

Thomas: Can we be more specific on the source of turbulence, which means some motion decaying? You talk about the hot stars as sources; long ago turbulence was believed to come from differential galactic rotation.

Colgate: I want to make sure that we know what we are discussing. In electromagnetic or magnetic turbulence, a charged particle, if displaced, will oscillate back and forth through some equilibrium point. This oscillation is a unique property of all turbulences associated with charged particles and a magnetic or electrostatic field. In gas dynamic turbulence, there is no such thing as an equilibrium value, unless you should be speaking of sonic turbulence. But almost always one speaks of turbulence in gas as being an arbitrary displacement of a particle, with only a small chance that the particle returns to the same point. This interpretation primarily means, in terms of physics, that you can have a persistent function like an eddy, which has no a priori reason to unwind. The distinction is decisive for the description of the two forms of turbulence. The aerodynamic turbulence is only poorly described by waves; but in the plasma case, we speak of $k$-values and waves and the modes of waves. Now first we have the sort of turbulence discussed so far, which has very short wavelengths, of the order of the Debije length (meters, or kilometers). Second there is hydromagnetic turbulence with energy input at $1 / k$ of the order of $100 \mathrm{pc}$, as discussed by Parker.

Parker: I think it is an oversimplification to say that the main input is the RayleighTaylor instability at $100 \mathrm{pc}$. It is certainly one of the inputs. I think non-uniform rotation of the Galaxy, plus the stirring of hot stars and sporadic local production of cosmic rays must be added, too. The Rayleigh-Taylor instability, together with the 
thermal instability, causes the gas to condense into clouds; and the hot stars and cosmic ray production tend to blow the clouds apart.

Colgate: I disagree with the differential rotation idea which leads to the concepts of two-dimensional turbulence. In two-dimensional turbulence, the eddies always progress to larger size.

Parker: You can develop three-dimensional turbulence in a one-dimensional shear, such as the non-uniform rotation. Therefore, I see no justification for saying that the non-uniform rotation cannot produce turbulence.

Colgate: Do you think that pure differential rotation alone with no heat input, as in the Earth's atmosphere, gives rise to turbulence?

Parker: I do not know. But of course, we do not have the shear alone; we have all the other heating, stirring, and agitation, which takes energy out of the non-uniform rotation in the form of turbulence.

Field: From the point of view of gas dynamics, can one characterize the turbulence spectrum of the plasma oscillations by some mean values which can then be put into the magnetohydrodynamic equations? For example, we might use a turbulent pressure which would be important in discussing shock propagation. [This question remained unanswered during part of the discussion. Finally Syrovat-skii brought it up again. Ed.]

Syrovat-skii: I should like to make a remark on the question by Field. The smallscale plasma turbulence gives us a new method of dissipation of large-scale motions. A good example is given by the solar flares. If we calculate the time of dissipation by the usual collisional processes, we obtain, say, a hundred or a thousand years for the dissipation of sunspot magnetic fields. But when we observe a solar flare, we see the field changing radically in a few minutes. Such a short time scale is brought about by small-scale plasma processes. Field's question is therefore how one effectively includes the small-scale plasma processes in the macroscopic description of interstellar gas dynamics.

Tsytovich: I can answer this question. Let us consider, for instance, ion-sound turbulence, or anomalous resistivity. One can introduce effective collisions that depend on the energy of the turbulence. They are very similar to normal collisions, in the sense that the dependence on the velocity is the same. This leads to a description of turbulent resistivity. However, we have no generalization of this procedure available. In some cases you cannot introduce effective collisions because the dependence on the velocity is not the same. Another question is: can one treat the turbulence as an external force in the magnetohydrodynamic equations? Work on this problem has been started, and it should be continued, I think.

Kaplan: There is a direct answer to Field as regards shock waves. All plasma turbulence processes in shock waves have dimensions less than the mean free paths. Thus these processes are beyond the scope of hydrodynamics and are therefore not included in hydrodynamic equations. Also, the energy density of plasma turbulence is usually much smaller than the density of thermal energy. But, if effective acceleration of particles in shock-wave turbulence is really taking place, these particles remove 
energy from the shock. This phenomenon must be taken into account in the RankineHugoniot equation. The energy of flow of plasma across the shock front is not conserved, and such shocks resemble the well-known shock with radiation.

Mestel: Do I take it correctly that acceleration of fast particles is not an important energy sink for dissipation of large-scale turbulent energy?

Parker: If you look at the damping processes both for very large-scale, hundred-pc disturbances in interstellar space and for small-scale things, which you might call plasma turbulence, it seems to me that the major dissipation known is not cosmic rays. It is diffusion and Landau damping. That is, the energy does not seem to go into the cosmic rays. The other side of the coin is that the energy which goes into cosmic rays is five or ten times as much as is available to the interstellar medium. If anything, it is the cosmic rays which drive the interstellar medium, rather than vice-versa.

Tsytovich: We know that the Galaxy is moving through the intergalactic medium. The velocity of this motion is about $100 \mathrm{~km} \mathrm{sec}^{-1}$, which is of the order of the thermal velocity of the electrons. Can this motion of the Galaxy be a source of turbulence excitation?

Colgate: Is not the density of the Metagalaxy $n \approx 10^{-5} \mathrm{~cm}^{-3}$ ? With $n$ for the Galaxy of the order of $1 \mathrm{~cm}^{-3}$, the ratio of densities excludes, I think, the excitation of either waves or turbulence of a very sizeable magnitude in the Galaxy.

Tsytovich: The excitation depends on the nature of the instabilities. For a plasma instability, the density ratio of $10^{-5}$ must be multiplied by the plasma frequency $\left(10^{4} \mathrm{sec}^{-1}\right)$; thus the growth time is $10^{-1} \mathrm{sec}^{-1}$. Only $10 \mathrm{sec}$, therefore, would be needed to excite turbulence. The kinetic energy of the inflow is enough to produce the cosmic rays.

Menon: From the observer's point of view the energy input is in the form of high velocity motions of small bits of matter and it goes ultimately into random motions of 20 to 100 pc clouds. The fact that the kinetic energies agree is not enough. We really must consider the question of the energy transformation.

Colgate: That comment is probably correct. But Tsytovich's question implied that the plasma turbulence is excited by the wave friction of the Galaxy moving through metagalactic space. One should ask first: is there sufficient energy in the collision alone? Then, after that question is answered, we should look at the wave field that would be excited. And the wave field that might be excited might have the finestructure scale that you are asking for.

Pikel'ner: If the Galaxy moves in the intergalactic gas with a rather high velocity, it would form a stationary shock wave in front of it. The thickness of this shock would depend on the plasma instability. Behind the front, there should be a layer with excited plasma turbulence. This front can also accelerate cosmic rays. [In his Report Spiegel, p. 201, discusses a related problem. Ed.]

Colgate: We now direct the discussion toward the subject of collisionless shock waves.

Kaplan: One of the most important points in the theory of the collisionless shock is the width of the shock. I would like to present a new argument that the width is of the 
order of an ion Larmor radius. The instability of plasma inside the shock front is usually connected with an ion-distribution function with two maxima, as was demonstrated earlier by Mott-Smith and by Tidman. This leads to excitation of plasma turbulence with low-frequency modes as follows. As in strong shocks, the inequality $T_{e} \gg T_{i}$ is fulfilled (for instance, by heating the gas in front of the shock by electron conductivity or by radiation); and we may expect powerful ion-sound turbulence (see the Report by Kadomtsev and Tsytovich, p. 108). Usually the first excited waves are those with wave numbers of the order of $V_{s} / \omega_{p i}$ where $V_{s}$ is the velocity of the shock and $\omega_{p i}$ is the ion-plasma frequency. Then, by the non-linear process of scattering on ions, the ion-sound waves decrease their wave numbers and frequencies until they reach their lower limit. If the frequency of ion-electron collisions is really very small, the lowest possible frequency is the gyrofrequency of ions $\omega_{B i}$, since at that frequency strong damping of ion-sound waves occurs. The shock front thickness will be of the order of the free path of the wave with smallest wave number; in this case, it is approximately $10 V_{s} / \omega_{B i}$. In the interstellar medium this quantity is about $10^{10}$ or $10^{11}$ $\mathrm{cm}$. If in a particular situation there is no magnetic field at all, or if the ion-electron collisions are somewhat more frequent, the smallest possible wave number is determined by other factors. One of the models appropriate for solar conditions was elaborated by a former student of mine (Zaitsev, 1967). He found the value for the thickness of the shock front to be $n_{e}^{1 / 2} \lambda_{D}^{5 / 2}$, where $n_{e}$ is the electron density and $\lambda_{D}$ is the Debije radius for the plasma after the shock. By coincidence, the same value is found for the shock thickness in the interstellar medium. Therefore, it seems to me that the value of $10^{10}$ to $10^{11} \mathrm{~cm}$ is a realistic estimate of the width of the shock front in the interstellar medium. This value is many orders of magnitude smaller than the value of $10^{15}$ to $10^{17} \mathrm{~cm}$ found if only the mean free path of ions and atoms is used. (Kaplan, S. A. and Pikel'ner, S. B.: 1963, The Interstellar Medium, Moscow (translation: 1970, Harvard University Press, Cambridge); Zaitsev, V. V.: 1967, Astron. Zh. 44, 490 (translation: Soviet Astron. 11, 392).)

Colgate: We have heard from the theoreticians that collisionless shock waves exist in plasmas and in the interstellar medium. I want now to bring up the question: is there any evidence, in the Galaxy or in the laboratory, that the collisionless shock really exists?

Davis: Space observations of the bow shock of the earth and of other shocks in free space should remove completely any doubt about the existence of collisionless shock waves.

Colgate: Is there any estimate of shock thickness or of the dissipation mechanism in the satellite experiments of the bow shock?

Davis: One can make estimates of the thickness, but it is not easy because the shock is not stationary. In principle you sail your spacecraft through the shock and see how long it takes. The problem is that the shock is moving rapidly relative to the spacecraft. The thickness is clearly small. The experiments cannot easily distinguish between a thickness of the order of the proton gyroradius and a thickness of the order of the geometric mean of the proton and electron gyromagnetic radii. The dissipation mechanisms are not clear, but strong electric fields are involved. 
Parker: We have much detailed evidence on the behavior of collisionless shocks in the solar system, and there are observations showing that fast particles are often generated copiously in these shock waves. One would conclude, scaling in a very crude way, that the much more vigorous blast waves from novae and supernovae must certainly generate a great many fast particles through plasma turbulence mechanisms.

Van de Hulst: I want to repeat that spatial resolution in astrophysical observations is not good enough to observe widths of less than $10^{15} \mathrm{~cm}$ anywhere in interstellar space. Only by indirect evidence can one conclude that collisionless shocks exist.

Podgornii: In the Institute of Space Research in Moscow, Dubinin, Managadze and I have tried to construct collisionless shock waves in the laboratory. In our experiments the parameters were: velocity $V: 3 \times 10^{7} \mathrm{~cm} \mathrm{sec}^{-1}$; density $n: 10^{13} \mathrm{~cm}^{-3}$; electron temperature $T_{e}: 15$ to $20 \mathrm{eV}$; ion temperature $T_{i}: 5 \mathrm{eV}$; and $B$ (frozen in the plasma) $40 \mathrm{G}$. These parameters were chosen to make the shock similar to those in the solar wind. For example, this laboratory plasma flow has the same Mach number as the solar wind, with respect to the Alfvén velocity and the magneto-sound velocity. The free path of the particles is 10 times larger than the size of the experiments. The ratios $\beta=8 \pi n k T / B^{2}$ and $\omega_{p i} / \omega_{B i}$ are comparable in the laboratory and in the solar wind, where $\omega_{p}$ and $\omega_{B}$ are the plasma frequency and the cyclotron frequency, respectively. The magnetic Reynolds number was about $10^{4}$. In the experiment we obtained collisionless shocks with a length of about $c / \omega_{p i}$, where $\omega_{p i}$ is the plasma-ion frequency and $c$ the velocity of light. We tried to determine how the energy was dissipated. One of the theories of collisionless shocks assumes instability of Alfvén and magneto-sound waves in the shock front. Under this assumption one should observe magnetic field fluctuations at a frequency near the ion Larmor frequency and the dimension of the fluctuations should be about $c / \omega_{p i}$. We measured such fluctuations by means of magnetic probes. One probe was placed upstream where there were almost no magnetic fluctuations. But in the shock and behind the shock, the magnetic fluctuations increased. By placing two probes in or behind the shock, we can estimate the lengths of the fluctuations from the measured correlation function.

A typical oscillogram of the fluctuations is presented in Figure 3. The upper trace shows the change of the magnetic field strength in the upstream probe. The second trace shows the output of the probe placed in the shock front. Figure 3 shows oscillograms from two downstream probes, when the distance between the two probes was less than $3 \mathrm{~cm}$. A rather good correlation of signals is seen. The correlation coefficient of the signals from the two probes as a function of their distance is shown in Figure 4. The half-width of the curve gives the correlation length of the fluctuations, which turns out to be, indeed, $c / \omega_{p i}$. In the region of interaction between the plasma flow and the magnetic field, $c / \omega_{p i}$ equals the ion Larmor radius. Measurements of the magnetic fluctuations have not been made previously. It is an interesting point that the spectra of the fluctuations around $\omega_{p i}$ are the same in our experiments and in space $\left[P(\omega) \propto \omega^{-3}\right]$. The experiments indicate that dissipation of the energy of the plasma flow arises from the instability of magneto ion sound or Alfvén waves, induced probably because of the 

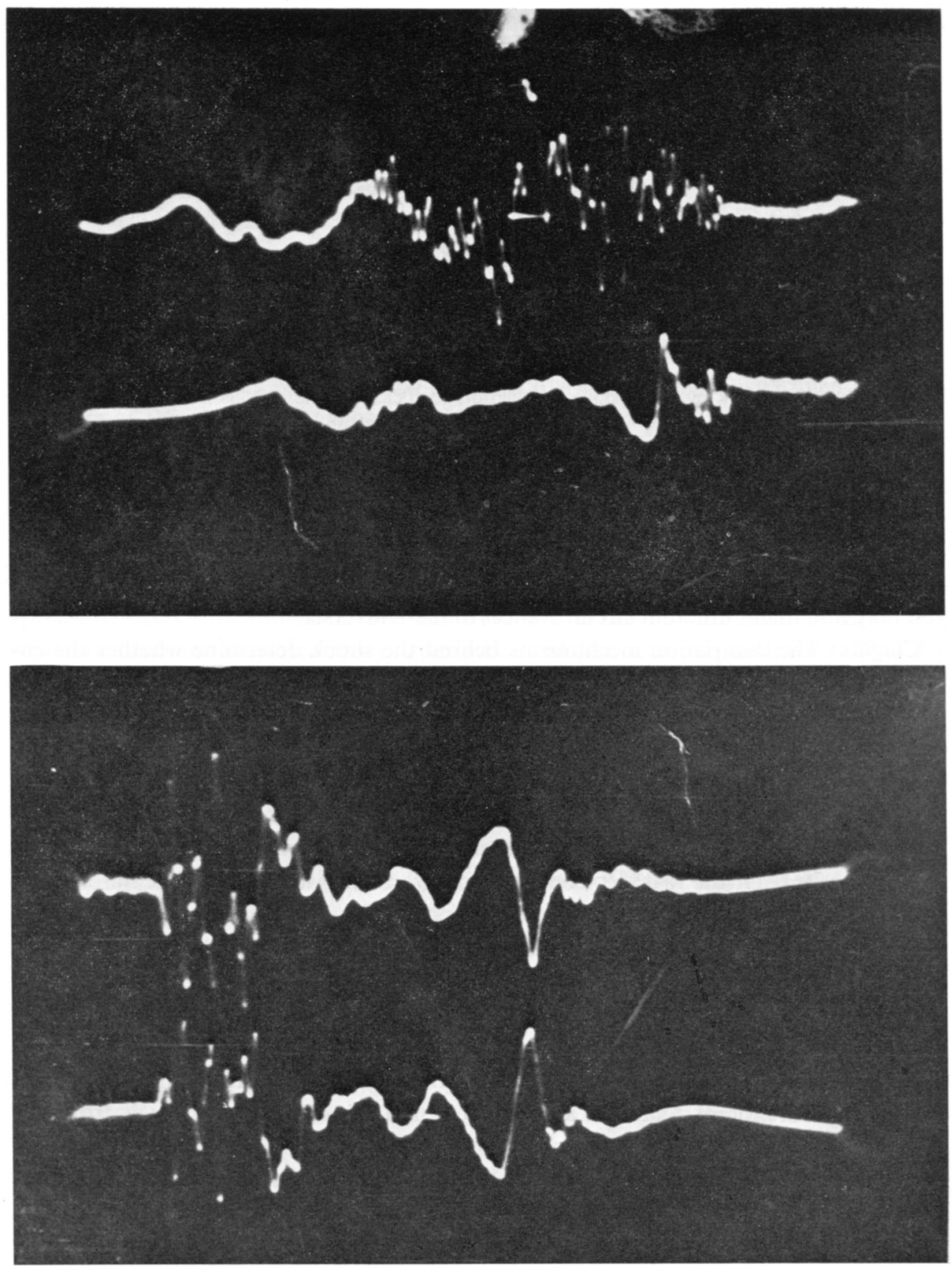

Fig. 3. (See the remark by Podgornii.) Oscillograms of magnetic-field fluctuations observed in laboratory experiments on the solar wind. 
anisotropy of the plasma pressure in the magnetic field, as discussed by Kadomtsev and Tsytovich (this volume, p. 108).

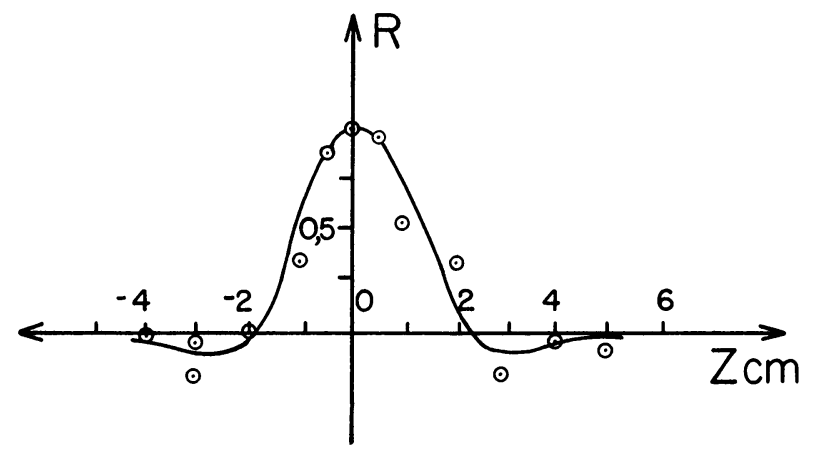

Fig. 4. (See the remark by Podgornii.) Correlation coefficient of the signals from two probes as a function of their distance.

Parker: There is one point which has been demonstrated in many investigations of the problem. Depending on the various dimensionless numbers that characterize any particular collisionless shock, there are many different interactions which may dominate the scene; and therefore, there are many different shock structures which you may find under different circumstances in the Universe.

Colgate: The dissipation mechanisms behind the shock determine whether the entropy is generated in ion waves or in electron waves, and therefore whether the energy goes into high energy electrons or into high energy ions. If enough energy goes into electrons, we might hope to see radio emission.

Kaplan: I have said previously that the plasma turbulence pockets can be seen, if there is an anisotropic distribution of fast electrons. In very strong shocks we may have such a distribution due to the acceleration of particles inside the shock. I am not sure, however, that the fast electrons can achieve relativistic energy when crossing a shock front thickness of small dimensions. Finally, I hope that the law of changing of the velocity of the shock during the passage through a medium with varying electron density $\left(V \propto n_{e}^{-1 / 4}\right)$ (Kaplan, 1967) is also valid for collisionless shocks. (Kaplan, S. A.: 1967, Astron. Zh. 44, 384 (translation: Soviet Astron. 11, 302).)

Field: Is it true that the final result of the generation of plasma turbulence is to increase the temperature far downstream from the main shock front? Therefore, are the Rankine-Hugoniot relations applicable as long as one is far enough from the shock front, because complete thermalization has occurred?

Colgate: Yes, the Rankine-Hugoniot relations are always fulfilled. The decay lengths behind the shock are associated with the excitation of additional degrees of freedom as the plasma approaches thermal equilibrium. What are the widths of waves coming out of the neutron star in the Crab?

Woltjer: The width of the waves in the Crab is of the order of $0.01 \mathrm{pc}$.

Colgate: If the particles are relativistic, the wavelength is small compared to the thermalization length. 
Zel'dovich: To answer Field's questions: At large distances there will be an equilibrium due to the relaxation of all the established processes. But near the front, there are several velocities at any one point. Instead of one Maxwellian distribution, there are several $\delta$-functions. However, for pressure and density this effect is not very important; therefore, a very exact Rankine-Hugoniot equation exists at a distance which is not necessarily very large. Very good laboratory experiments on the widths of shocks with transition from a wide shock to a normal shock are made by Sagdeev, who is not only a theoretician, but also has recently become an experimentalist. I would like Dr. Karpman, who has worked with him, to comment.

Karpman: There was a series of experiments in Novosibirsk, performed by the Sagdeev group in the last few years in which detailed structures of collisionless shocks were investigated. If in a rather cold plasma a shock front exists perpendicular to a magnetic field, then, as was predicted theoretically and observed experimentally, the structure of the collisionless shock is of an oscillatory kind, with the value $c / \omega_{p e}$ for the dimension of one oscillation. The decay length depends on the nature of the dissipation. If the dissipation from the turbulence is of the collective type, then several large-scale oscillations appear. The oscillatory structure appears only if the angle $\theta$ between the normal to the shock front and the direction of the magnetic field is less than $\sqrt{ }\left(m_{e} / m_{i}\right)$. If the angle is larger, the size of one oscillation is $\lesssim\left(c / \omega_{p l}\right) \theta$. All this applies to cases with rather small Mach numbers. If the Mach number is large, the structure becomes non-oscillatory, aperiodic, and like a usual [collisional [?] Ed.] shock structure. The length or thickness in this case is approximately the Larmor radius. The ions in the front are rotating around the magnetic field. All these types of shocks were observed experimentally in special tubes with different geometry for the magnetic fields.

Podgornii: I want to compare the Sagdeev experiment and the experiments I described before (p. 144). The principal difference consists in the conditions of the experiments. In the Sagdeev experiment, a low-density plasma was used; the ratio $\beta=$ $8 \pi n k T / B^{2}$ was much less than unity. But in our experiments, where we tried to simulate the solar wind, a high-density collisionless plasma was employed. We have $\beta$ somewhere near unity. In our case, there is no regular magnetic structure, only microfluctuations. In a recent issue of Usp. Fiz. Nauk, Sagdeev and I have described all these cases and compared the results of all different experiments.

Colgate: I should like to mention a problem associated with the power law spectrum of cosmic rays at low energies. Observationally we obtain the low-energy cosmicray spectrum by correcting for the interplanetary modulation. Theoretically we have predictions based on the various models for a supernova explosion. In both cases we seem to obtain a smooth curve down to very low energies. The difficulty, from the standpoint of collisionless shock wave theory and the interaction with the interstellar medium, is that this power law does not give you anywhere a sharp discontinuity in the velocity spectrum. All the excitation mechanisms of instabilities along the lines of force require such a discontinuity and are therefore excluded. Only the component of the explosion which is across the local magnetic field should lead to a collisionless 
shock and then only when the energy densities of the particles have been reduced to a value of $\beta$ of the order of unity. This implies that the explosion will blow a hole in the magnetic field of the Galaxy, of the order of $100 \mathrm{pc}$ in dimension, before the stress of the particles in the hole becomes comparable to the field. These predictions, both the one about the direction perpendicular to the field and the one parallel, disagree with the observations. This forms a problem that has worried me for many years, and I wonder if anyone here knows the solution.

Field: McKee at Berkeley has been considering collisionless shock-fronts in a relativistic twinstream situation. Thus far his computer studies indicate that an instability will develop and, presumably, also a shock; I cannot speak, however, on your specific point that the gradual build-up of particles may preclude such a shock.

I want to bring up a question connected with collisional shock fronts in neutral gas. I wonder if the presence of a small ionization of, say, one per cent will significantly change the properties of the shock waves. I assume that the shock fronts move so slowly that they do not ionize the gas. Will there be a major change? It does not seem so to me.

Pikel'ner: In a shock front in a partially ionized gas, ions have a collective instability and form a sharp front. Neutral atoms do not have a collective instability, and they can form only collisional shocks. Fifteen years ago I calculated the structure of shocks in a partially ionized gas (Pikel'ner, 1954). There are several layers, a sharp shock for ions and a thick layer in which there are interactions (especially charge exchange interactions) between ions and neutral atoms. In this thick layer the densities of ions and atoms change gradually. But under the usual conditions in the interstellar gas, even the thickness of the thick layer should be less than can be observed.

I should like to add another possible observation on plasma phenomena. For instance, there are some filamentary nebulae which are shock waves. To calculate their emission, you have to know the electron temperature immediately behind the fronts; but the distribution of energy between electrons and ions is dependent on the kinds of instability which transform the energy of shock waves into the thermal energy of particles. From a classical point of view, this shock energy should go to the ions and from a plasma point of view, it should go to the electrons. But the emission of the filament depends very strongly on whether the ions or the electrons happen to receive the shock energy. Quite generally, plasma effects are important for dissipation of magnetic fields in neutral lines. Usually in cold interstellar space, plasma turbulence will be very weak. Only in some very violent places in the Galaxy, for instance in the central parts or in shock waves going out from supernovae, are there conditions favorable for high energy plasma turbulence and for the acceleration of fast particles. (Pikel'ner, S. B.: 1954, Izv. Krymsk. Astrofiz. Observ. 12, 94.) (A discussion in English of this work may be found in Sections 9 and 10 of Interstellar Gas Dynamics by S. A. Kaplan, Pergamon Press, Oxford, 1966.)

Spiegel: Since you are really trying hard to find an observable collisionless shock, may I raise the possibility that we look for such things in stellar systems? For example, 
if the spiral arms are waves, they are narrower than a stellar mean-free path; that result would be suggestive of a collisionless shock.

Colgate: The term collisionless shock pretty well implies the concept that there exists an enhanced dynamic friction over and above the $r^{-2}$ force law, independent of whether it is gravitational or electrostatic force. The collisionless shock occurs when you take a large number of particles of one kind of charge and have them interact in a cooperative way with a large number of particles of the opposite sign. The collective interaction may be either through electrostatic forces or through magnetic forces. But in any case, the idea is that the collective interaction is stronger. The shocks that you get in stellar systems ought to be called collisional shocks.

Spiegel: I am speaking of waves which involve only collective interaction.

Parker: The difficulty with the stars is, as Colgate said, that you do not have opposite charges to give overall neutrality. Therefore, whenever you have two systems falling through each other, there is always a large potential well, and the characteristic length for instability (the interaction between the two oppositely streaming components of stars) is always comparable to the dimensions of the components of the system itself. You never can get evidence for a clean-cut shock.

Field: I think Spiegel is quite correct. There is a plasma-like mode in a stellar system for which the dispersion relation is: $\omega^{2}=\omega_{g}^{2}+k^{2} c^{2}$ with $\omega_{g}^{2}=4 \pi G \varrho$. The point is that, instead of having a real plasma frequency, you have an imaginary one corresponding to the attractive forces of gravitation. The analogue of a plasma oscillation is then the Jeans instability.

The characteristic length, $\lambda_{J}\left(=2 \pi c / \omega_{g}\right)$ associated with such a shock might be the Jeans length, corresponding to the Debije length in the plasma shock case. However, it must be kept in mind that the dispersion relation is derived from moment equations. One finds from the Boltzmann equation that waves with $k \gg k_{J}=\omega_{g} / c$ are heavily Landau damped and therefore do not propagate as readily as the dispersion relation would suggest. I am not sure what the effect of the damping would be on the formation of a shock. I would take issue with Parker. While it is rigorously true that the Jeans length tends to be of the order of the size of the system, one can think of a special system that is quite interesting, for example, a rotating disk supported by stellar motions (pressure) in the vertical direction and by centrifugal force in the plane. The radius can be far greater than the Jeans length. A spiral galaxy is such a system. The Jeans length in the stars of the Galaxy is about $100 \mathrm{pc}$; it is conceivable, therefore, that we could have a shock with a thickness of about $100 \mathrm{pc}$, still much smaller than the radius of the system, $10^{4} \mathrm{pc}$.

Pikel'ner: If the stellar system is rotating, then the coriolis forces are important and play the role of the magnetic field in the plasma. This was studied in detail by Marochnik. 